\title{
La acción social comunitaria de las asociaciones de mujeres inmigrantes en tiempos de crisis
}

\author{
Community social action of migrant women's associations \\ in a time of crisis
}

Trinidad L. Vicente Torrado • trinidad.vicente@deusto.es

DEUSTUKO UNIBERTSITATEA/UNIVERSIDAD DE DEUSTO

Amaia Unzueta Sesumaga • kambotik@gmail.com

KAMBOTIK CONSULTORÍA SOCIAL

Recibido: 04/04/2016

Aceptado: 01/07/2016

\section{Resumen}

Este artículo analiza la realidad socioeconómica y familiar de las mujeres inmigrantes en el País Vasco y, sobre todo, trata de visibilizar su propia acción colectiva y las distintas formas en que están dando respuesta a las situaciones de necesidad a las que tienen que hacer frente, derivadas de sus proyectos migratorios y de la actual coyuntura de crisis. Empleando una metodología cualitativa (basada en entrevistas en profundidad) este artículo trata de exponer el papel de las asociaciones de mujeres inmigrantes desde la perspectiva de la acción social comunitaria, así como el valor de su auto-organización como vía necesaria para el cambio hacia una sociedad inclusiva y equitativa.

A pesar de su débil estructura organizativa, estas asociaciones proporcionan apoyo psicológico y social, orientación y capacitación laboral, espacios culturales y de disfrute del ocio y del tiempo libre, espacios para la conciliación de la vida familiar y laboral, pequeñas iniciativas de autoempleo, sensibilización en torno a cuestiones migratorias y/o de género, etc. E, incluso, se convierten frecuentemente en la puerta de entrada para sus socias a la red pública y privada de servicios sociales. Estas asociaciones son, en definitiva, recursos clave en la acción social comunitaria, por lo que habrá que tenerlas en cuenta en la planificación de políticas e intervenciones, y habrá que apoyarlas en su proceso de fortalecimiento asociativo, de modo que podamos contar con ellas como agentes válidos para la coordinada acción social.

Palabras clave: Mujeres inmigrantes, crisis, asociacionismo, comunidad, trabajo social

\section{Abstract}

This article analyzes the socio-economic and family situation of women immigrants in the Basque Country. It mainly focuses on their collective action and the different ways they use to face the difficulties derived from their migration projects and their living conditions at the present time of crisis. Using a qualitative methodology (in depth interviews), this article highlights the role of women immigrants' associations from the perspective of the community social action, and the value of their self-organization as a necessary way to the change towards an inclusive and equitable society.

Despite of their weak organizational structure, these associations offer psychological and social support, work orientation and training, cultural programs and leisure activities, spaces for the conciliation of family and work, small self-employment initiatives, activities to increase the sensitivity on migration and/or gender issues, etc. Even, they become very often the entrance door for their members to the public and private network of social services. Thus, these associations are key resources in the community social action. So, it is necessary to keep them in mind in the process of planning policies and interventions, and it is also necessary to support them in their process of strengthening in order to be able to count on them as important agents for the coordinate social action.

Key words: Female immigrants, crisis, associations, community, social work 


\section{INTRODUCCIÓN}

La presencia de población de origen extranjero en el País Vasco no ha descendido a pesar de la crisis económica, aunque en todo caso, su peso relativo en la sociedad vasca $(8,4 \%)$ permanece todavía bastante alejado del alcanzado a nivel estatal (13,2\%).

La inmigración internacional (184.187 personas a 1 de enero del 2015, según los datos del INE) ha tenido, sin duda, un importante impacto en la dinámica demográfica vasca, revirtiendo la tendencia a la pérdida de población que se venía registrando en el periodo previo a su llegada ${ }^{1}$. La inmigración ha contribuido, además, a su rejuvenecimiento, dada su inferior edad media y su ligeramente superior tasa de natalidad ${ }^{2}$. Precisamente la estructura demográfica de la población vasca va a ser clave para entender el papel de la inmigración femenina en esta sociedad, incluso en la actual coyuntura de crisis.

En este marco, este artículo trata de analizar la acción social comunitaria específica de las asociaciones de mujeres inmigrantes en el País Vasco, dado que las mujeres constituyen la mayoría de la población procedente de otros países en este territorio (95.199 frente a 88.998 hombres a comienzos del año 2015). Para ello, en primer lugar contextualizaremos la situación socioeconómica de las mujeres inmigrantes en la sociedad vasca, examinando su ubicación en el mercado de trabajo así como las principales estrategias migratorias desarrolladas tanto desde el punto de vista laboral como desde el punto de vista familiar. Y, posteriormente, nos centraremos en las asociaciones lideradas por estas mujeres, así como en las estrategias por ellas desarrolladas con el objeto de recuperar la dimensión comunitaria de la planificación e intervención social en contextos culturalmente diversos y con población en situaciones de necesidad socioeconómica derivadas del proceso migratorio y/o de la presente coyuntura generalizada de crisis.

Este artículo está basado en el análisis del trabajo de campo llevado a cabo por las autoras en el desarrollo de dos proyectos de investigación en los que se ha empleado una metodología cualitativa (entrevistas en profundidad a representantes de las asociaciones de mujeres inmigrantes registradas en el País Vasco)33. Concretamente se han mantenido entrevistas con representantes de las siguientes catorce asociaciones: Alnour, Amalur Charrua, Arahma ASEMA, Bidez-Bide, Djelia Taama Musoo, Garaipen, Grupo de Mujeres Senegalesas, Lacasiun, Mujeres en la Diversidad, Mujeres del Mundo Unidas, Mujeres Migradas de Getxo, Safa, y Voz-Colombia. A todas ellas les agradecemos desde estas líneas su inestimable colaboración.

\footnotetext{
1 Euskadi ha visto aumentar su población en 90.661 personas en los últimos quince años, debido a la inmigración internacional, que ha pasado de 35.143 personas en el año 2000 a 184.197 en el 2015. En este mismo periodo, en cambio, la población nacida en el País Vasco ha descendido en 58.393 personas.

${ }^{2}$ En el año 2014 un 14,8\% de los nacimientos fueron de una madre extranjera, a pesar de que estas mujeres apenas representan el 6,2\% de la población femenina total.

${ }^{3}$ Estos dos proyectos son: «Asociacionismo de mujeres inmigrantes en el País Vasco» y «Proyecto piloto de investigación-acción sobre género y codesarrollo en el País Vasco», este último financiado por el Gobierno Vasco.
} 


\section{LA INMIGRACIÓN FEMENINA Y SU INCORPORACIÓN AL MERCADO LABORAL}

La llegada de la inmigración internacional a Euskadi, registrada especialmente a lo largo de los últimos quince años, se inició coincidiendo con el último ciclo de crecimiento económico, en el que se produjo un notable aumento de la demanda laboral, así fuera en sectores de baja cualificación como el servicio doméstico o la construcción. A lo largo de los últimos ocho años, en cambio, la inmigración se ha hecho eco de la actual coyuntura de crisis económica y de empleo que afecta a España en general y al País Vasco en particular", por lo que ha disminuido el saldo migratorio. Aunque este resultado se debe no tanto al incremento de las salidas como a la drástica reducción en el número de entradas, que ya no responden tanto a nuevas personas llegadas en busca de trabajo sino más bien a los procesos de reagrupación familiar puestos en marcha por la población inmigrante venida hace ya unos años.

Durante el último periodo de bonanza económica, el mercado de trabajo vasco presentó una serie de características que explican en buena medida la creciente demanda de mujeres inmigrantes. El crecimiento del sector terciario, la segmentación de los mercados de trabajo y el incremento de la flexibilidad y de los sectores informales van a dar lugar a una serie de nichos laborales reservados de forma particular a estas mujeres, especialmente en el sector del servicio doméstico y de los cuidados personales, cuando no en la industria del sexo (Vicente, 2006); todos ellos caracterizados por su precariedad y una escasa protección social y legal.

La creciente - aunque todavía relativamente reciente- incorporación de las mujeres autóctonas al trabajo extradoméstico en nuestra sociedad 5 , la todavía escasa participación de los hombres en la realización de las tareas del hogar y del cuidado 6 , así como la reducida implicación del Estado en la provisión de políticas sociales con las que hacer frente a esta situación (realidad que se ve agravada en la actual coyuntura económica y de debilitamiento del

\footnotetext{
${ }^{4}$ España es actualmente el país de la UE con un mayor nivel de desempleo (con 4.789.000 personas en paro, el 20,9\% de la población activa a finales del 2015). En el País Vasco, el paro también ha crecido a lo largo de los últimos años, si bien su incidencia (12,9\%) es claramente inferior a la media estatal. De hecho ésta es la Comunidad Autónoma con menor porcentaje de paro del Estado español.

${ }^{5}$ Las mujeres se han incorporado tardíamente al mercado de trabajo español en comparación con otros países de la UE. Ahora bien, para el año 2013 la brecha de género en las tasas de ocupación en España (9,2 puntos porcentuales), se situaba ya por debajo de la media de la OCDE (12,6), y no muy lejos de la registrada en los países escandinavos (donde se encuentra en torno a 5-7 puntos porcentuales). Para más información, puede consultase la página web del Instituto Nacional de Estadística (www.ine.es)

${ }^{6}$ Según la Encuesta de Presupuestos del Tiempo, 1993-2013 del Eustat, los hombres han incrementado en $43 \mathrm{~min}$. y las mujeres han reducido en $59 \mathrm{~min}$. como media su dedicación al hogar y la familia respecto al año 1993. Con todo, ellos apenas dedican la mitad del tiempo que las mujeres a la realización de estas tareas, a pesar de que disfrutan de 52 min. más diarios de tiempo de ocio y vida social (Sagastizabal y Luxán, 2015: 395396). Además, son los hombres desempleados quienes más tiempo dedican al hogar, por lo que habrá que comprobar si este comportamiento varía o permanece cuando cambie su situación laboral (Carrasco, 2013). Y es que, «la dinámica de evolución de la equidad no suele ser un proceso continuo, sino sujeto a movimientos de conflicto, de retroceso o de recomposición según los avatares económicos o el poder de los grupos implicados en el cambio» (Fernández, Artiaga y Dávila, 2013: 73).
} 
La acción social comunitaria de las asociaciones de mujeres... | Trinidad L. Vicente Torrado et al.

Estado de bienestar, con continuos recortes en los servicios sociales ofrecidos), han llevado a un buen número de familias a recurrir al exterior, concretamente a las mujeres inmigrantes, para lograr la conciliación de la vida laboral y familiar mediante la externalización de, al menos, parte de las labores domésticas y de cuidado de personas dependientes (Martínez Buján, 2010; Acosta, 2015). Posponiendo además con ello la discusión del reparto de roles patriarcales en el seno del hogar y de la sociedad. Esta transferencia del trabajo de cuidado, que funciona como una cadena global en la que participan sólo las mujeres (Ehrenreich y Hochschild, 2003), viene a poner en evidencia, además, las crecientes desigualdades entre las mujeres y cómo en el trabajo de reproducción social se entrecruzan las diferencias de género, nacionalidad, clase social, étnia, etc. (Parella, 2003; Carrasco, 2013).

La migración y el trabajo, por tanto, no siempre suponen una mejora en la condición de las mujeres, puesto que el contrato que da lugar a la sociedad civil y al Estado es un contrato social (capitalismo), un contrato sexual (patriarcado) y un contrato de esclavitud (supremacía de la raza blanca sobre otras razas y etnias, eurocentrismo, xenofobia, etc.) (Pateman, 1995).

Los diferentes roles asignados a hombres y mujeres migrantes en la sociedad van a explicar la feminización de los flujos migratorios hacia el País Vasco, puesto que estos nichos de empleo en los sectores más bajos de la escala laboral son trabajos considerados típicamente femeninos, abandonados en la medida de lo posible por las mujeres autóctonas debido a la escasa valoración social, baja remuneración y reducidas oportunidades de movilidad laboral o social ascendente por ellos ofrecidas. Y ello se va a producir aún cuando los niveles educativos de la mayoría de las mujeres inmigrantes son muy superiores a los exigidos para su desempeño: más de la mitad de este colectivo (58\%) cuenta con estudios secundarios e, incluso, universitarios, superando incluso los niveles educativos alcanzados por los hombres inmigrantes (49\%), quienes en cambio vienen mostrando una mejor y más variada incorporación al mercado laboral vasco (Martín, 2012).

Pero el trabajo femenino no sólo goza de menos prestigio social que el masculino y está peor pagado, sino que disfruta de menor protección legal, se realiza sin contrato en la mayoría de los casos y carece de normativas que garanticen que se desempeña en condiciones aceptables ${ }^{7}$. Todo lo cual va a poner permanentemente en cuestión su capacidad para

\footnotetext{
7 En el año 2011, la OIT aprobó normas laborales internacionales destinadas a reconocer el trabajo doméstico retribuido como un empleo similar al resto, y a mejorar las condiciones de trabajo de las empleadas domésticas. De igual manera, en España, el país de la UE con mayor número de empleadas de hogar, se aprobaba en ese mismo año el Real Decreto 1620/2011, de la Regulación laboral del servicio del hogar familiar, en un intento de lograr una modificación de la relación laboral especial del servicio doméstico equiparándola de forma progresiva con el régimen general. Esta nueva regulación incorpora algunos avances, como un mayor acceso a protecciones y derechos sociales; pero tampoco ha cubierto todas las expectativas ya que, aunque integrada en el régimen general, sigue constituyendo un sistema especial que no reconoce, por ejemplo, la prestación por desempleo, dejando en una situación de extrema vulnerabilidad a las personas inmigrantes en este sector que pierden su trabajo, especialmente si durante ese tiempo en el que se encuentran desempleadas tienen que renovar además su permiso de residencia. Por otra parte, según el Gobierno Vasco, de las 90.000 mujeres que se estima trabajan en este sector, apenas 26.000 se encontraban afiliadas en diciembre del 2012, un 65\% más que un año antes (Galende, 2013). La mayoría de las trabajadoras del servicio doméstico continúan, por tanto, en la
} 
garantizar su propia subsistencia económica y la de sus familias, la obtención y/o la renovación de los preceptivos permisos para residir regularmente en esta sociedad, su derecho a la reagrupación familiar, etc.

Esta segmentada oferta laboral dirigida a las mujeres migrantes en base a las identidades de género viene a mostrar cómo a pesar de los avances en el reconocimiento —al menos a nivel teórico - de los derechos humanos de las mujeres y de la equidad de género, éstos quedan diluidos por la intervención de los mercados de trabajo que, en los países receptores al igual que en los países emisores, siguen circunscribiendo la participación laboral femenina a puestos relacionados con sus tradicionales roles reproductivos, asignados con independencia de su cualificación profesional, limitando en buena medida sus oportunidades de promoción laboral, de reconocimiento social, de participación socio-política... en definitiva, de integración social en el sentido más amplio del término. A pesar de todo lo cual muchas de estas mujeres van a valorar su experiencia migratoria como positiva, en la medida en que contribuye a la mejora de las condiciones de vida familiares y a la ampliación de las oportunidades especialmente para sus hijos e hijas.

\section{ESTRATEGIAS MIGRATORIAS DE LAS MUJERES INMIGRANTES EN TIEMPOS DE CRISIS}

Si la incorporación laboral de las mujeres inmigrantes se caracterizaba por la precariedad ya con anterioridad a la llegada de la crisis económica que nos viene azotando a lo largo de los últimos años, la actual coyuntura socio-laboral no ha hecho sino empeorar la situación. La crisis económica ha tenido un gran impacto en el conjunto de la población, con un notable aumento del desempleo y de la precarización de las condiciones laborales. Ahora bien, este negativo impacto es todavía más acusado entre la población inmigrante y, especialmente, entre sus mujeres, debido a su diferenciada - y en la mayoría de los casos desigual y desequilibrada- posición en el acceso a los recursos económicos, en el reparto de tiempos y trabajos o en el acceso a los espacios de poder (Gálvez y Rodríguez, 2011).

La crisis mundial está teniendo consecuencias muy negativas para las mujeres: si al comienzo de la misma el desempleo masculino creció por encima del femenino, especialmente en los países más desarrollados, la brecha de género mundial en las tasas de desempleo ha aumentado a lo largo de los últimos años, siendo superior que la registrada a comienzos del siglo xxI (OIT, 2012).

Y a similares conclusiones han llegado distintos estudios centrados en el contexto español (Colectivo Ioé, 2011; Gálvez y Rodríguez, 2011; Juliano, 2012), cuestionando la opinión com-

economía sumergida, y su salida a la luz no se presenta como una tarea sencilla (García Sanz, 2011). Además, menos de la mitad de las mujeres extranjeras en alta laboral en el País Vasco disfruta de un contrato indefinido (49\%), siendo por otra parte un contrato a tiempo parcial en la mayoría de los casos (52\%). 
partida por amplios sectores de la sociedad que apunta que las mujeres son las menos perjudicadas por el impacto de la actual coyuntura socioeconómica (Gabinete de Prospección Sociológica, 2013): en el año 2009 el salario medio anual de las mujeres autóctonas en el Estado español era casi una cuarta parte inferior al percibido por los hombres del mismo origen; el de los hombres inmigrantes apenas superaba la mitad del de los varones autóctonos, mientras que el de las mujeres de origen extranjero apenas alcanza un 42,9\% (Colectivo Ioé, 2011). Una brecha salarial que, lejos de reducirse, parece venirse ensanchando a lo largo de los últimos años (INE, 2015).

Las mujeres inmigrantes en la sociedad vasca registran, por otra parte, una tasa de desempleo claramente inferior a sus compatriotas varones (18 y 29\% respectivamente), aunque muestran asimismo un mayor crecimiento en su tasa de inactividad a lo largo de los últimos años (31\% frente al 17\% de los hombres inmigrantes) (Martín, 2012). Esto no obstante, las distintas nacionalidades de origen presentan destacadas diferencias en estas tasas. Las mujeres procedentes de algunos países latinoamericanos (como paraguayas o bolivianas) muestran, por ejemplo, unas tasas de actividad superiores a la media, mientras que otros colectivos femeninos africanos (como el marroquí o el senegalés) se caracterizan por unas crecientes tasas de actividad así como por unas elevadas tasas de desempleo, que llegan a alcanzar el 50\%. Datos que reflejan cómo el creciente deseo de incorporarse al mundo laboral de estas mujeres, movilizadas muchas de ellas ante la situación de desempleo del hasta ahora proveedor principal de la familia, corre en paralelo al incremento de sus tasas de paro.

La crisis económica está obligando a un creciente número de mujeres procedentes de otros países, inactivas hasta ahora, a incorporarse al mercado laboral; un mercado laboral que en la actual coyuntura socio-económica está agravando aún más si cabe la división sexual del trabajo, así como la precariedad en las condiciones laborales. En este contexto, muchas mujeres se están viendo obligadas a rebajar sus expectativas y a aceptar peores condiciones de trabajo, renunciando a empleos más acordes con sus niveles de formación y a una movilidad laboral ascendente, cuando no accediendo incluso a una movilidad social descendente (Juliano, 2012), y renunciando al recurso del autoempleo, debido a los crecientes obstáculos al acceso a créditos bancarios.

Además, a lo largo de los últimos años las mujeres inmigrantes no han visto descender su demanda laboral en el sector doméstico, en respuesta a la intensificación de la carga de trabajo en el hogar así como de los trabajos de cuidado de personas dependientes, como consecuencia de la crisis y de los recortes gubernamentales en las políticas públicas de igualdad y en los sistemas de protección social (Moreno y Bruquetas, 2011). Un trabajo reproductivo que, tanto de forma no remunerada como de forma remunerada, tiende a ser patrimonio del género femenino (Fernández, Artiaga y Dávila, 2013), por el bajo nivel profesional, escasa remuneración y reducida valoración social que recibe, así como por su poca utilidad para la promoción profesional futura (Acosta, 2015). Unas características que hacen poco probable que los hombres se interesen por él, al igual que muchas mujeres que van a poder optar por una mejor inserción laboral. 
La crisis está significando una reprivatización de la reproducción social (Carrasco, 2013), y es que las crisis se superan con una intensificación del trabajo remunerado y, sobre todo, del no remunerado, de las mujeres (Gálvez y Rodríguez, 2011). La actual coyuntura económica está limitando las posibilidades de contar con una empleada doméstica por parte de muchas familias, lo cual está provocando a su vez una reducción a la baja de las ya de por sí precarias condiciones laborales en este sector. Y en este contexto, serán precisamente las mujeres inmigrantes, quienes con mayores dificultades de acceso al mercado laboral y con menores recursos, se vean obligadas a desempeñar estos trabajos, así sea en condiciones de semiesclavitud, en un intento de continuar con sus proyectos migratorios y los de sus familias.

Pero la crisis económica no sólo va a tener un impacto en las estrategias laborales de las mujeres inmigrantes, sino que también lo va a tener en sus estrategias familiares. En primer lugar, el endurecimiento de las condiciones de empleo y la frecuente reducción de los salarios van a obligar a muchas mujeres inmigrantes a asumir mayores cargas de trabajo remunerado, así sea a costa de un elevado coste personal, y/o a reducir el monto de las remesas enviadas a sus familiares en origen (Vicente, Ruiz y Unzueta, 2011).

Por otra parte, muchas de estas mujeres van a ver aumentado su trabajo de cuidados y sus problemas de conciliación de la vida familiar y laboral, especialmente aquellas que cuentan con hijos e hijas pequeños y que carecen de una red extensa de apoyo en la sociedad de acogida. Los drásticos recortes del gasto público en prestaciones sociales, en servicios educativos o sanitarios, así como la reducción de los ingresos del hogar debido al endurecimiento de las condiciones laborales, cuando no al desempleo de alguno de sus miembros, están suponiendo un nuevo traspaso y aumento de la carga de tareas para los hogares, al no poder hacer frente al pago de la externalización de estos servicios (ej. comedores escolares, plazas de guardería, ludotecas, colonias infantiles en periodos vacacionales...). Este aumento de tareas, especialmente intenso en el caso de las familias con menos recursos, como son las inmigrantes, va a recaer principalmente sobre las mujeres, incluso cuando éstas también desempeñan un empleo remunerado. Todo lo cual va a condicionar en buena medida el uso que ellas hacen de su tiempo, aumentando asimismo las tensiones dentro del hogar por la dificultad que supone la gestión de los cuidados (Carrasco, 2013; Juliano, 2012). De esta manera, la incorporación de las mujeres al mercado laboral no implica en muchos casos sino el fortalecimiento de los roles tradicionales, así como un aumento de las tareas a desempeñar por ellas (doble jornada).

En este contexto, no son pocas las familias inmigrantes que desearían reagrupar a algún ascendiente, para convivir con esa persona y contar, además, con una ayuda en el desempeño de estas tareas de cuidado en el hogar. Deseos que chocarán con las numerosas restricciones impuestas por la legislación española al respecto, así como con las crecientes dificultades para cumplir con los requisitos económicos y de vivienda exigidos para tal fin. Exigencias estas últimas que también van a obstaculizar, cuando no impedir, la reunificación familiar del cónyuge o de descendientes en origen, y que siempre han sido especial- 
mente complicadas de satisfacer por parte de las mujeres inmigrantes dadas sus condiciones laborales y/o residenciales.

Por otra parte, ante esta coyuntura socio-laboral, muchas familias inmigrantes van a optar por la desagrupación familiar; es decir, por la re-emigración de alguno de sus miembros en paro hacia otros destinos en búsqueda de empleo, o por el retorno de menores que, de otro modo, quedarían desatendidos ante las dificultades de conciliación o por considerar inferior el coste de su educación y/o mantenimiento en origen. En la actual coyuntura de crisis, los retornos de muchas personas adultas responden a la necesidad de enfrentar el desempleo, mientras que en el caso de los hijos e hijas ya no responden sólo a procesos de reagrupación poco armoniosos sino a la necesidad de reorganizar el trabajo reproductivo de cuidados (Pedone, 2013). Una tarea que nuevamente recaerá de forma primordial en mujeres, en esta ocasión ubicadas en las sociedades de origen (Vicente, Ruiz y Unzueta, 2011).

Analizados los principales impactos de la crisis económica en las mujeres inmigrantes afincadas en el País Vasco, así como los principales cambios en sus estrategias laborales y familiares, vamos a centrarnos a continuación en la respuesta asociativa por ellas impulsada en la actual coyuntura.

\section{EL ASOCIACIONISMO EN EL TRABAJO SOCIAL COMUNITARIO}

La inmigración internacional es uno de los procesos sociales que, desde la década de los '90, más retos ha planteado y sigue planteando al Trabajo Social. Uno de ellos es, sin duda, la necesidad de recuperar la dimensión comunitaria de la planificación e intervención social en contextos diversos culturalmente y con población en situaciones de necesidad socioeconómica derivadas del proceso migratorio. De hecho, el trabajo en y con la comunidad sigue siendo el nivel de intervención más desatendido en la práctica profesional del Trabajo Social (Martínez y Peña, 2010). Entre las cuestiones a abordar desde el Trabajo Social en materia de inmigración, encontramos tanto las de tipo social y económico como las de tipo cultural, las cuales requieren de una acción social que contribuya a la gestión constructiva de las diferentes identidades para la convivencia. Esta acción no puede limitarse únicamente al plano individual y familiar, sino que debe orientarse y abrirse hacia la comunidad con el objetivo de hacer partícipes a los grupos concernidos del cambio social necesario. Y es que la participación de las personas y de las comunidades en la resolución de las problemáticas que les afectan, y en su propio proceso de desarrollo y de cambio social es uno de los principios básicos del trabajo social comunitario. Para llevarlo a cabo, Marchioni (1989) señala que, como mínimo, deben actuar tres agentes que son: (a) el cuerpo político responsable de las administraciones, (b) el equipo profesional y el personal técnico de los servicios públicos o de los privados, y (c) la población y sus organizaciones sociales. Lo ideal es que la acción social comunitaria gire alrededor de las relaciones entre 
estos tres agentes, aunque no siempre es posible por la pluralidad de posiciones y puntos de vista.

Nuestro interés en abordar el asociacionismo de las personas inmigrantes radica en la idea de que la auto-organización y la participación en y desde las organizaciones comunitarias son clave para el cambio social (Abdelaziz, Cuadros y Gaitán, 2004; Marchioni, 2004). Unas organizaciones sociales sólidas, participativas y representativas de los intereses y necesidades de la comunidad resultan esenciales para llevar a cabo un trabajo social que integre la dimensión colectiva: «la comunidad organizada es el primero y principal de los recursos existentes» (Marchioni, 1989: 54).

En el ámbito de la inmigración, existen organizaciones sociales de apoyo a la población inmigrante y organizaciones de personas inmigrantes. En los apartados siguientes, vamos a centrarnos en las segundas, en aquellas creadas y sostenidas principalmente por población inmigrante, y concretamente por mujeres, en el País Vasco con el fin de visibilizar su propia acción colectiva y la forma en que ellas mismas están dando respuesta a situaciones de necesidad derivadas de sus proyectos migratorios y de las condiciones de acogida y asentamiento que ofrece la sociedad vasca, especialmente en este contexto de crisis y de recortes sociales en el que las asociaciones vuelven a convertirse, en muchos casos, en los únicos recursos de apoyo existentes y en espacios de lucha y resistencia.

\section{ASOCIACIONISMO DE MUJERES INMIGRANTES EN EL PAÍS VASCO}

En el País Vasco, el asociacionismo inmigrante de origen extranjero empieza a configurarse con el cambio de siglo. Una década después, existen unas 153 asociaciones de inmigrantes (Maza, 2013), de las cuales 16 son asociaciones de mujeres (Unzueta y Vicente, 2011) ${ }^{8}$. Destacamos el dato numérico como prueba de que existe un movimiento de mujeres que responde a necesidades e intereses distintos a los que habitualmente se abordan desde los espacios mixtos y que, por lo tanto, también conviene tenerlas en cuenta en la planificación de acciones sociales dirigidas tanto a ellas a modo de colectivo, como al conjunto de la comunidad con la que conviven.

Efectivamente, el hecho de que hayamos optado por focalizar nuestra atención en la acción colectiva de la población inmigrante femenina se explica por dos factores que, a nuestro entender, siguen vigentes. Por un lado, continua existiendo un sesgo androcéntrico en los estudios sociales que tiende a obviar el papel activo de las mujeres en los procesos sociales; y, por otro, dicho sesgo hace que predomine una visión asistencialista hacia la po-

\footnotetext{
${ }^{8}$ Consideramos como asociación de mujeres inmigrantes aquélla que ha sido creada y está formada por mujeres, y en la que la mitad de su Junta Directiva o al menos la mitad de sus socias son mujeres de origen extranjero.
} 
blación inmigrante femenina que acaba por condicionar el tipo de políticas e intervenciones que se diseñan para ellas como grupo social. En este sentido, y en coherencia con la propuesta de rescatar el trabajo social comunitario, consideramos que es útil conocer primero cuáles son las principales características de sus organizaciones, para después plantear en qué medida pueden ser tenidas en cuenta como recursos comunitarios para la intervención social y/o en qué haría falta apoyarlas.

Se trata de asociaciones con escaso recorrido (con una media de constitución formal de siete años), que tienden a organizarse según grandes grupos geográficos (mujeres del Magreb, de Latinoamérica, africanas...) y, además, acogen entre sus socias a mujeres autóctonas. Funcionan principalmente con voluntariado y, a menudo, el liderazgo y gestión asociativa recae en reducido grupo de personas (entre dos y tres). Esta cuestión de los recursos humanos y formas de participación es clave a la hora de analizar el nivel de fortalecimiento asociativo, ya que, en términos generales, se observa una participación discontinua y más bien ligada al disfrute de actividades puntuales. Esto, entre otros motivos, se explica por las dificultades que encuentran muchas mujeres para poder participar activamente, y que tienen que ver con la forma en que se da su proceso de inserción laboral, así como con las ya mencionadas mayores responsabilidades domésticas y de cuidados que ellas asumen en comparación con sus parejas.

Esta limitada y precaria inserción laboral que experimenta gran parte de las mujeres procedentes de otros países constituye al mismo tiempo un obstáculo y un motor para su participación social. La frecuente vulneración de derechos que tiene lugar en este sector y/o los obstáculos para encontrar trabajo hace que las mujeres busquen en las asociaciones un espacio en el que recibir apoyo psicológico y social, y es a raíz de esta búsqueda de ayuda y orientación que a menudo empieza a darse su participación asociativa. Más allá del terreno laboral, existen otros motivos del proceso migratorio que inducen a la creación de estos espacios como son la necesidad de contar con redes sociales para disfrutar del ocio y tiempo libre, para combatir la soledad y para hablar sobre la experiencia migratoria en sus diferentes dimensiones (personal, familiar, laboral, social) y ser escuchadas. En definitiva, existen factores asociados a la migración y a las oportunidades que ofrece el contexto de recepción que hacen que la diversidad existente entre el colectivo femenino inmigrante converja en espacios comunes desde los que afrontar las dificultades derivadas de su condición de mujeres e inmigrantes. De hecho, esta doble condición también ayuda a entender por qué han surgido tales asociaciones al margen de aquellas creadas por las mujeres autóctonas y de otras de inmigrantes mixtas (conformadas por hombres y mujeres).

Con respecto a estas últimas, recordemos que el género como categoría social determina las diferentes experiencias de migración de hombres y de mujeres, lo cual conlleva que unos y otras acaben por tener necesidades e intereses distintos en su proceso migratorio y que esto, por lo tanto, afecte al tipo de actividades que se quieren llevar a cabo desde una asociación. Dado que en la amplia mayoría de las asociaciones mixtas predomina una cultura organizacional basada en valores patriarcales, hay una tendencia a que predomine 
la voz masculina frente a la femenina en la toma de decisiones, quedando las demandas de las mujeres relegadas a un segundo plano. Este es, en consecuencia, uno de los factores que motiva a las mujeres a organizarse entre ellas.

Las diferentes agendas asociativas y formas de funcionamiento establecidas por las asociaciones de mujeres feministas autóctonas son razones que, por otra parte, explican que haya mujeres inmigrantes que hayan optado por constituir sus propias asociaciones. Lo que no significa que estén cerradas a la colaboración y al trabajo en red. En este sentido, una temática que puede acercar a las asociaciones de mujeres, inmigrantes y autóctonas, es el de los cuidados, puesto que su abordaje supone, entre otros aspectos, cuestionar las desigualdades de género que mantienen la división sexual del trabajo dentro y fuera de los hogares. Todo ello muy necesario en la compartida lucha por la igualdad.

La colaboración y el trabajo en red ya ha comenzado, sobre todo entre las propias asociaciones de mujeres inmigrantes, siendo este un reto por el que algunas han apostado a través de Kafeminista; una red surgida en Bizkaia con el objetivo de realizar incidencia política sobre los temas que les preocupan, de negociar con administraciones públicas y otros agentes sociales acciones que favorezcan mejores condiciones de vida en mayor justicia e igualdad, y de construir y consolidar una red de mujeres inmigrantes con una visión crítica del mundo frente a una realidad capitalista, patriarcal, desigual e injusta (Ulloa y Carranza, 2012).

Este tipo de iniciativas, orientadas al trabajo coordinado entre diferentes organizaciones sociales, como es el caso de las conformadas por mujeres inmigrantes, son recursos clave en la acción social comunitaria, bien para tenerlas en cuenta en la planificación de políticas e intervenciones, bien para apoyarlas en su proceso de fortalecimiento organizativo que permita contar con ellas como agentes válidos para la acción social coordinada.

\section{ESTRATEGIAS SOCIALES Y POLÍTICAS DE LAS ASOCIACIONES DE MUJERES INMIGRANTES}

A menudo, las asociaciones se convierten en la puerta de entrada a la red pública y privada de servicios sociales, puesto que ofrecen un primer espacio de acogida en el que las mujeres se sienten más cercanas y cómodas para hablar sobre su situación vital. Desarrollan así una labor de apoyo desde la solidaridad y desde el compromiso de querer contribuir a mejorar las condiciones de vida de las mujeres, y sirven de puente para derivar casos a otros agentes de la red coordinada de servicios sociales cuando la situación lo requiere. Por una parte, gran parte de estas asociaciones están realizando un trabajo social no profesionalizado que responde a necesidades prácticas de las mujeres y que se traduce en actividades, por ejemplo, de información y orientación sobre los recursos del entorno, 
ocio y tiempo libre, apoyo psicológico y emocional, etc. Por otra, unido a estas actividades de tipo práctico, algunas van desarrollando un trabajo político de fondo que se refleja en actividades como talleres de formación y de sensibilización sobre cuestiones migratorias, laborales, de género, etc. cuyo fin es concienciar sobre las desigualdades estructurales que condicionan los proyectos de vida de las mujeres. La línea divisoria entre lo social y lo político es difusa, puesto que el disfrute por parte de las mujeres de acciones sociales de tipo más práctico puede derivar, con el tiempo o simultáneamente, en una participación en actividades de trasfondo más político como son los talleres de sensibilización o acciones reivindicativas de calle. En cualquier caso, la realidad es que el contexto general de crisis está teniendo un impacto en la estabilidad del proyecto migratorio de muchas mujeres que, a su vez, tiene su reflejo en el tipo de actividades que las asociaciones priorizan llevar a cabo con los menores recursos económicos de los que disponen. En general, se focalizan más los esfuerzos en necesidades de tipo práctico ante el aumento de situaciones de mayor necesidad y riesgo de exclusión social.

Al igual que existe una gran diversidad entre la inmigración femenina que nos obliga a descartar un patrón único de mujer inmigrante, existen también variedad de planteamientos y actividades entre las asociaciones de mujeres inmigrantes en el País Vasco. Y es que, aunque hay situaciones comunes que se comparten como mujeres e inmigrantes, también hay factores diferenciales que determinan la experiencia migratoria en destino, como son el origen étnico, los valores culturales de referencia, el nivel educativo, la trayectoria personal, profesional y asociativa antes de migrar, el nivel de conciencia sobre las desigualdades de género y sobre las desigualdades estructurales en general, etc. Por ello, a continuación vamos a referirnos a algunos ejemplos de acciones que se están realizando según las diferentes dimensiones del proceso migratorio (familiar, laboral y socio-cultural), sobre las que introduciremos algunas consideraciones.

\subsection{La dimensión familiar}

La dimensión familiar es un espacio vital y esencial de los procesos migratorios que, a menudo, se obvia en las políticas y planes sobre la materia porque corresponde a la esfera privada, es decir, al ámbito de los afectos y de los cuidados. Por ejemplo, el caso de la migración laboral de las mujeres latinoamericanas que lideran el proyecto migratorio familiar, quedando el resto de miembros de la familia en origen (descendientes, pareja, etc.), coloca a las mujeres en una situación nueva de tener que desarrollar las relaciones familiares en la distancia. La gestión más o menos positiva de estas relaciones dependerá de cada mujer y sus circunstancias. No obstante, desde esa asignación diferenciada de roles e identidades que sigue colocando sobre las mujeres la responsabilidad de los cuidados estén donde estén, ellas viven con mayores sentimientos de culpa, en comparación con los hombres, el ejercicio de su maternidad en la distancia, así como las dificultades que puedan surgir en las dinámicas familiares transnacionales. Otros casos relativos a la dimensión familiar son los que conllevan situaciones de reagrupación familiar o, como se está viendo a raíz de la crisis, de des-agrupación familiar cuando los miembros que habían sido 
reagrupados, principalmente menores, tienen que retornar al país de origen por la precaria situación socio-económica de la familia. Todos estos cambios en las dinámicas familiares afectan el día a día de las mujeres y su proceso de integración en la sociedad de destino, de ahí que sea importante prestar atención a esta dimensión en las intervenciones de trabajo social.

Entre las asociaciones de mujeres inmigrantes, encontramos el caso de la Asociación Bidez Bide, que lleva a cabo un trabajo de intervención familiar con mujeres y hombres inmigrantes tanto en situaciones de familias transnacionales como en situaciones de reagrupación y desagrupación, en coordinación con otros recursos comunitarios especializados en la temática. Es interesante observar cómo su práctica asociativa contribuye a llamar la atención sobre la necesidad de abordar la dimensión afectivo-familiar del proceso migratorio, apoyando a sus socias en los momentos de soledad y ayudándoles a prepararse psicológicamente para los reencuentros con los familiares —especialmente con la proletras largos periodos de separación.

\subsection{La dimensión laboral}

Esta es otra de las dimensiones esenciales del proceso migratorio que, como hemos destacado anteriormente, está claramente atravesada por las desigualdades de género. La migración laboral de la gran mayoría de las mujeres, ligada en la mayoría de los casos al sector servicios y más concretamente al desempeño del trabajo doméstico y de cuidados, se produce en circunstancias habitualmente precarias e, incluso, frecuentemente en el ámbito de la economía sumergida. Una mayor precariedad que, tal y como se ha detallado en apartados anteriores, influye en las diferentes experiencias migratorias de mujeres y de hombres, siendo más difícil para ellas la estabilización de su proyecto migratorio individual y/o familiar.

Frente a esta realidad, las asociaciones también despliegan sus estrategias de apoyo y de emprendimiento en busca de vías alternas a la precariedad. Como estrategias de apoyo, varias de ellas organizan cursos de formación básica dirigidos a mejorar la capacitación profesional de las mujeres en cuestiones para las que tienen cabida en el mercado de trabajo (cursos para la obtención del carnet de manipulación de alimentos, cursos sobre cuidado de personas dependientes, etc.). Y también hay quienes llevan a cabo talleres y charlas informativas sobre la normativa del servicio doméstico y de cuidados, la vulneración de derechos en el sector, etc.

En relación con las estrategias de emprendimiento, hemos observado iniciativas a nivel micro que permiten a la asociación y a las mujeres implicadas en ellas obtener unos pequeños ingresos a nivel colectivo e individual, pero que en ningún caso conforman grandes proyectos que representen una salida laboral para las mujeres. Hablamos de iniciativas como las emprendidas por la Asociación Mujeres en la Diversidad para la prestación profesional de servicios de catering y/o de guardería. Más allá del valor económico que 
puedan tener, es interesante resaltar el papel sensibilizador de estas iniciativas en sí mismas como, por ejemplo, el servicio de guardería, a través del cual se constata la necesidad de atender a las dificultades de conciliación de la vida familiar y laboral. En este sentido, dicha Asociación plantea la falta de apuestas por el desarrollo de recursos solidarios para la provisión de los cuidados como una estrategia de acción entre organizaciones de la sociedad civil que complementen las escasas medidas de conciliación desarrolladas a nivel público.

\subsection{La dimensión socio-cultural}

Entre las múltiples cuestiones del proceso migratorio dentro de esta dimensión vamos a destacar dos que están siendo abordadas desde algunas asociaciones de mujeres.

La primera de ellas es la relativa al analfabetismo de las mujeres de habla no castellana en su lengua de origen y en la lengua del lugar de destino. Por ej., la Asociación de $\mathrm{Mu}-$ jeres Árabe Musulmanas-Safa constata que las mujeres analfabetas procedentes de países del Magreb tienen grandes dificultades para aprender el castellano o el euskera y, por lo tanto, para su integración, situándolas en una situación de vulnerabilidad al no disponer de las herramientas básicas para aprender la/s lengua/s de destino que les permitan desenvolverse con autonomía. Ante esta realidad y los obstáculos que algunas mujeres encuentran para poder acceder a recursos públicos de enseñanza de las lenguas de la sociedad de acogida, como son las Escuelas para Personas Adultas, en los últimos años esta Asociación viene organizando cursos de aprendizaje del castellano dirigidos a mujeres, a la vez que reclama mayores apoyos públicos para el aprendizaje de la legua de origen: el árabe. Y es que no cabe duda de la necesidad del conocimiento de la lengua de la sociedad de acogida para favorecer la integración de las personas inmigrantes, pero es asimismo importante el reconocimiento de la lengua de origen, ya que ésta no sólo conforma la principal vía de comprensión y expresión de las personas, sino que es, además, el medio de identificación entre los miembros de una comunidad de pertenencia y, por otra parte, su buen conocimiento y manejo favorece el aprendizaje de un segundo idioma (Unesco, 2003).

La otra cuestión sobre la que queremos llamar la atención tiene que ver con la apuesta que varias asociaciones hacen por el trabajo hacia una toma de conciencia sobre cómo el género, como categoría social, construye la identidad de mujeres y de hombres y determina los proyectos de vida de unas y de otros. No todas las asociaciones lo plantean de la misma forma ni parten de los mismos niveles de análisis y empoderamiento individual y colectivo; sin embargo, lo interesante está en observar los procesos de cambio personal que esto conlleva y los beneficios que trae para un mayor bienestar de las mujeres. Entre las actividades realizadas en esta línea están los talleres y encuentros grupales en los que, a partir de la propia experiencia personal, se promueve la reflexión desde la mirada de género, utilizando metodologías participativas y dinámicas. 


\section{CONCLUSIONES}

Las mujeres inmigrantes conforman uno de los grupos sociales más afectados por la actual coyuntura económica desfavorable, debido a su precaria incorporación laboral y a las enormes dificultades para conciliar trabajo productivo y reproductivo; especialmente en unos momentos en los que se hace necesario, además, hacer frente a una intensificación tanto del trabajo remunerado como, sobre todo, del no remunerado, con el objeto de responder a las necesidades familiares.

La actual crisis socioeconómica constituye un obstáculo y, al mismo tiempo, un motor para la participación social de las mujeres inmigrantes en el movimiento asociativo por ellas mismas conformado. Estas asociaciones, caracterizadas por su juventud, débil estructura organizativa y planificación a corto plazo (debido a las enormes dificultades que encuentran sus socias para participar en ellas activamente) proporcionan, no obstante, apoyo psicológico y social, orientación y capacitación laboral, espacios culturales y de disfrute del ocio y del tiempo libre, espacios para la conciliación de la vida familiar y laboral, pequeñas iniciativas de autoempleo, sensibilización en torno a cuestiones migratorias, de género, etc. dirigida al conjunto de la sociedad, reivindicando un trato más igualitario... E, incluso, se convierten frecuentemente en la puerta de entrada para sus socias a la red pública y privada de servicios sociales.

Estas asociaciones son, en definitiva, recursos clave en la acción social comunitaria, por lo que habrá que tenerlas en cuenta en la planificación de políticas e intervenciones, y habrá que apoyarlas en su proceso de fortalecimiento asociativo, de modo que podamos contar con ellas como agentes válidos para la coordinada acción social.

\section{REFERENCIAS BIBLIOGRÁFICAS}

Abdelaziz, M., Cuadros, A. y Gaitán, L. (2004). La intervención social con colectivos inmigrantes. Madrid: Universidad Pontificia de Comillas.

Acosta, E. (2015). Cuidados en crisis. Mujeres migrantes hacia España y Chile, Bilbao: Universidad de Deusto.

Carrasco, C. (2013). El cuidado como eje vertebrador de una nueva economía. Cuadernos de Relaciones Laborales, 31 (1), 39-56.

Colectivo Ioé (2011). Nota sobre los efectos socioeconómicos de la crisis para la población inmigrada. Papeles, 113, 85-95.

Ehrenreich, B. y Hochschild, A.R. (eds.) (2003). Global woman: Nannies, maids, and sex workers in the new economy, Nueva York: Henry Holt \& Co. 
Fernández, C., Artiaga, A. y Dávila, M.C. (2013). Cuidados, género y transformación de identidades, Cuadernos de Relaciones Laborales, 31 (1), 57-89.

Gabinete de Prospección Sociológica (2013). Sociómetro Vasco 52. La crisis y el mercado laboral, Gobierno Vasco. Recuperado el 18 de junio de 2013 de http://www.euskadi.net

Gálvez, L. y Rodríguez, P. (2011). La desigualdad de género en las crisis económicas. Investigaciones Feministas, 2, 113-132.

Galende, J.L. (2013). Entran en vigor mañana los últimos cambios sobre las empleadas de hogar. El Correo. 31 de Marzo.

García Sanz, C. (ed.) (2011). Inmigrantes en el servicio doméstico, Madrid: Talasa.

Instituto Nacional de Estadística (INE) (2015). Encuesta anual de estructura salarial. Serie 2008-2012, Recuperado el 18 de marzo de 2016 de http://www.ine.es

Juliano, D. (2012). Género y trayectorias migratorias en épocas de crisis. Papers, 97 (3), 523540.

Marchioni, M. (2004). La acción social en y con la comunidad. Zaragoza: Certeza.

- (1989). Planificación social y organización de la comunidad. Alternativas avanzadas a la crisis. Madrid: Editorial Popular.

Martín, M.J. (2012). La mujer inmigrante en la CAPV 2010, Bilbao: Ikuspegi.

Martínez Buján, R. (2010). Bienestar y cuidados. El oficio del cariño: Mujeres inmigrantes y mayores nativos, Madrid: CSIC.

Martínez, E. y Peña, L. (coord.) (2010). III Jornada de Trabajo Social. REDefiniendo el trabajo comunitario. Vitoria: EUTS-UPV/EH.

Maza, S. (2013). Las asociaciones de migrantes de origen extranjero en el País Vasco. Un análisis sobre su participación en el espacio público. Cuadernos Bakeaz, 105.

Moreno, F.J. y Bruquetas, M. (2011). Inmigración y estado de bienestar en España, Barcelona: La Caixa.

OIT (2012). Global employment trends 2012, Ginebra: Autor.

Parella, S. (2003). Mujer, inmigrante y trabajadora: la triple discriminación. Barcelona: Anthropos. 
Pateman, C. (1995). El contrato sexual. Barcelona: Anthropos.

Pedone, C. (2013). Familias que trascienden fronteras. Estrategias de retorno de migrantes procedentes de Ecuador y Colombia, en C. Pedone y S. Gil (eds). Políticas públicas, migración familiar y retorno de la población latinoamericana en Cataluña (pp. 33-41), Barcelona: CIIMU.

Sagastizabal, M. y Luxán, M. (2015). Género y uso del tiempo, en M. Legarreta (coord.). Dos décadas de cambio social en la C.A. de Euskadi a través del uso del tiempo. Encuesta de presupuestos del tiempo. 1993-2013 (pp. 385-423), Donostia-San Sebastián: Eustat.

Ulloa, E. y Carranza, C. (2012). Kafeminista, Revista de Mujeres del Mundo-Munduko Emakumeak, 53. Recuperado el 21 de marzo de 2013 de http://es.scribd.com/ doc/115772456/53

UNESCO (2003). La educación en un mundo plurilingüe. París: Autor.

Unzueta, A. y Vicente, T.L. (2011). Asociacionismo de mujeres inmigrantes en el País Vasco: actuaciones y desafíos. Zerbitzuan, 49, 81-91.

Vicente T.L. (2006). Importancia de los flujos migratorios de mujeres, en C. Blanco (ed.). Migraciones. Nuevas movilidades en un mundo en movimiento (pp. 206-233) Barcelona: Anthropos.

Vicente, T.L., Ruiz, A. y Unzueta, A. (2011). Remesas, género y desarrollo. Las migraciones colombianas en el País Vasco. Bilbao: Bakeaz. 